Case Report

\title{
Treatment Resistant Severe Digital Ischemia Associated with Antiphospholipid Syndrome in a Male Patient with Systemic Sclerosis
}

\author{
Orhan Küçüikşahin,, ${ }^{1}$ Aşkın Ateş, ${ }^{1}$ Alexis K. Okoh, ${ }^{2}$ Emre Kulahcioglu, ${ }^{2}$ \\ Murat Turgay, ${ }^{2}$ and Gülay Kınıklı ${ }^{1}$ \\ ${ }^{1}$ Department of Rheumatology, Ankara University Medical Faculty, Sihhiye, 06410 Ankara, Turkey \\ ${ }^{2}$ Department of Internal Medicine, Ankara University Medical Faculty, Sihhiye, 06410 Ankara, Turkey
}

Correspondence should be addressed to Orhan Küçükşahin; orhankcs@hotmail.com

Received 22 May 2014; Accepted 27 June 2014; Published 8 July 2014

Academic Editor: Tsai-Ching Hsu

Copyright (C) 2014 Orhan Küçükşahin et al. This is an open access article distributed under the Creative Commons Attribution License, which permits unrestricted use, distribution, and reproduction in any medium, provided the original work is properly cited.

\begin{abstract}
We report the case of a male patient with limited cutaneous systemic sclerosis (SSc) that was complicated with severe digital ischemia, resistant to medical treatment. Due to the lack of treatment response, further laboratory and imaging studies were conducted. Findings were compatible with antiphospholipid syndrome and oral warfarin was added to the treatment regimen. After successful anticoagulation no further recurrences of digital ischemia were seen. An underlying etiology in SSc patients with treatment resistant digital ischemic necrosis should be suspected for accompanying antiphospholipid syndrome (APS).
\end{abstract}

\section{Case Report}

A 44-year-old, previously well, nonsmoker male presented with severe digital ischemia of multiple fingers. Six months ago, due to digital gangrene, his distal left index finger was amputated. On examination, digital necroses of the distal right index, right fourth, and left third fingers were seen (Figures 1(a) and 1(b)). Investigations revealed the following: ANA $(1 / 1000$ centromeric pattern, +++$)$ and anticentromere antibody were positive ( $1422 \mathrm{U} / \mathrm{mL}$; normal: $0-10 \mathrm{U} / \mathrm{mL}$ ) and erythrocyte sedimentation rate of $65 \mathrm{~mm} /$ hour, C-reactive protein of $92 \mathrm{mg} / \mathrm{dL}$ (normal: $0-3 \mathrm{mg} / \mathrm{dL}$ ), thrombocytes of 100000 (normal: $150-40010^{9} / \mathrm{L}$ ), and ANCA were negative. Nailfold capillaroscopy was consistent with an active scleroderma pattern (diffuse giant capillaries, microhemorrhages, and a reduction in number of capillaries). Chest radiograph, ECG, and HRCT findings of the thorax were normal. Esophagus motility test was normal as well. An upper extremity arterial Doppler ultrasound showed a normal flow pattern.

A diagnosis of limited cutaneous sclerosis was made. The patient was treated with acetylsalicylic acid $150 \mathrm{mg} /$ day, pentoxifylline $1200 \mathrm{mg} /$ day, nifedipine $60 \mathrm{mg} /$ day, $400 \mathrm{mg} /$ day hydroxychloroquine, and prednisolone $10 \mathrm{mg} /$ day. Despite this therapy, he had severe attacks of Raynaud's phenomenon and newly developed digital ischemic ulcers. Two months later intravenous (IV) iloprost, bosentan, high-dose steroid, and IV cyclophosphamide were added.

Due to the lack of treatment response further investigations were promptly done in order to define the underlying etiology of recurrent digital gangrene. Digital subtraction angiography (DSA) imaging of the upper extremity demonstrated occlusion of the left ulnar and right radial and ulnar arteries (Figure 2). Laboratory tests for APS showed LA: 1.4 (upper limit: 1.2) anti-Beta2 glycoprotein IgM: 9.3 MPL U/mL $(0-5)$, anticardiolipin IgM: 7.5 MPL U/mL (0-5), and thrombocytopenia. Triggered by the suspicion of an underlying possible infection, the patient was tested for syphilis, leptospirosis, Lyme disease, hepatitis $\mathrm{B}$ and $\mathrm{C}$ viruses, and human immunodeficiency virus (HIV); however results from infectious diseases panel were unremarkable. A diagnosis of an overlap syndrome, SSc, associated with antiphospholipid syndrome was made and oral warfarin was added to his treatment regimen. 


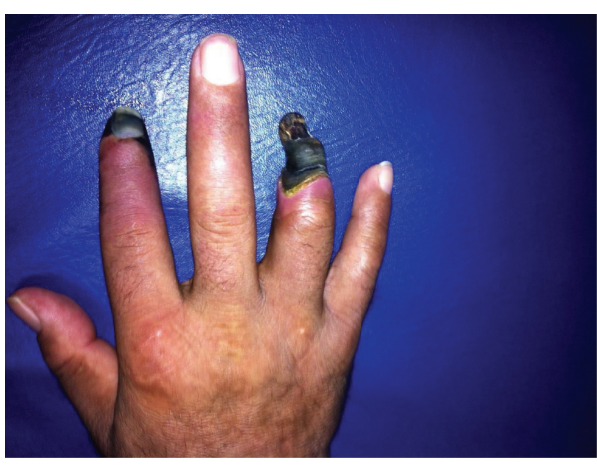

(a)

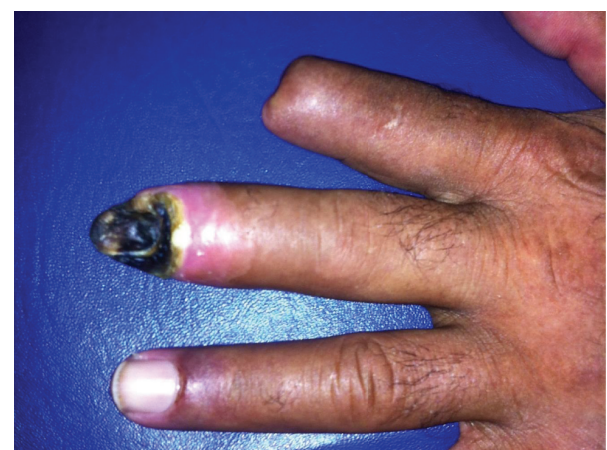

(b)

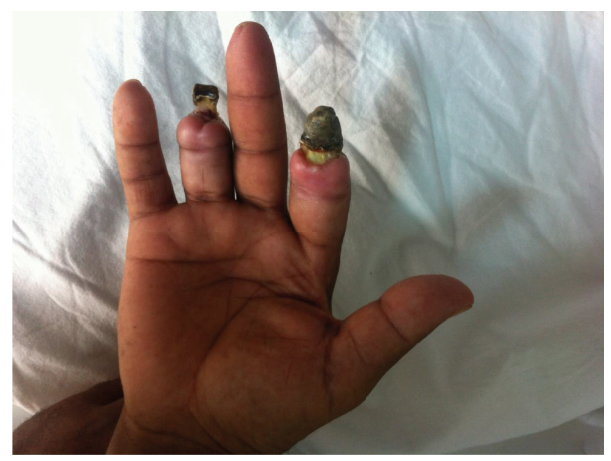

(c)

Figure 1: (a) Right hand showing digital gangrene on the index and fourth fingers, (b) left hand showing digital gangrene of third finger, and (c) six months after aggressive therapy.

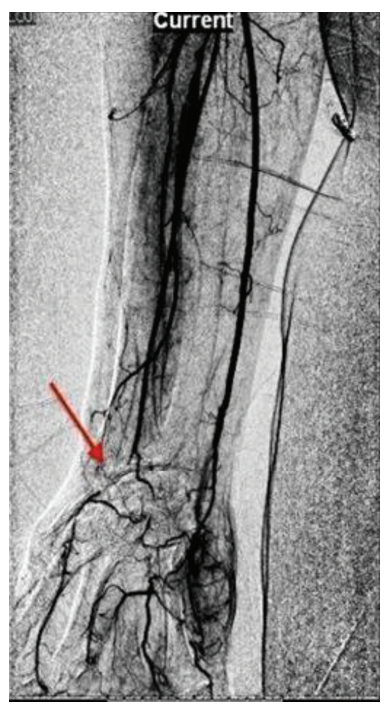

(a)

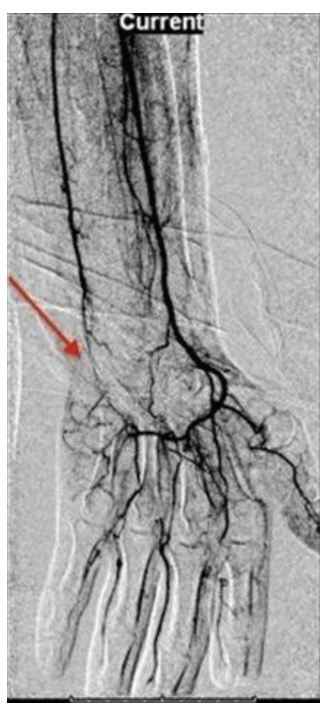

(b)

Figure 2: (a) Arrow showing total occlusion of the right radial and ulnar arteries at the wrist level. Filling of the radial artery seen after a short segment. (b) Arrow showing occlusion of the left ulnar artery at the wrist level with an open radial artery.

Capillaroscopy performed on his follow-up visit six months later revealed findings (right 2, 3, and 5 fingers; 2-3 non angiogenic capillaries; absent giant capillaries and microhemorrhages, left 5 and 6 fingers; and 5-6 microhemorrhages) consistent with late stage scleroderma.
On current treatment including oral daily use of bosentan, sildenafil, hydroxychloroquine, acetyl salicylic acid, warfarin, and monthly IV cyclophosphamide over six months of follow-up, the patient remained well, with no further recurrences of digital ischemic lesions (Figure 1(c)). 


\section{Discussion}

The coexistence of SSc/APS is a very rare overlap syndrome, found in less than $1 \%$ of scleroderma patients, yet the positivity for antiphospholipid antibodies in SSc is higher $[1,2]$. When it is seen, it is associated with more severe manifestations including digital infarct, gangrene, and pulmonary hypertension [3]. Minatani et al. previously reported the first presentation of this overlap syndrome in a male patient [4]. One case series with five female patients [5] and other three cases of female patients [6-8] were also reported in the literature.

Treatment with bosentan or its combination with sildenafil has been reported to decrease the number of new ulcers in systemic sclerosis $[9,10]$. Ambach et al. reported resolution of digital ulcers in a 73-year-old female patient who had uncontrolled disease over a 10 -year period after a combination therapy of bosentan and sildenafil [10].

Surprisingly, despite treatment with a range of immunosuppressive therapies and vasodilators, this patient's digital ischemia continued to progress. The patient was treated with methylprednisolone and cyclophosphamide considering the fact that small vessel vasculitis potentially contributed to the microangiopathy. Blood pressure and renal function were monitored carefully for the risk of scleroderma renal crisis. Despite the combination of various therapeutic agents, over 2 months, we failed to halt the progression of digital ischemia.

After reconsideration of the causes of underlying recurrent, treatment resistant digital gangrene, an overlap syndrome, SSc, associated with antiphospholipid syndrome was diagnosed with further laboratory and imaging studies; oral warfarin was added to the treatment regimen. Inevitably, the infarcted tissue was amputated, but over a year of followup, the patient remained well, with no further recurrences of digital ischemic lesions.

\section{Conflict of Interests}

The authors declare that they have no conflict of interests.

\section{Acknowledgment}

The authors would like to thank Actelion Pharmaceuticals Ltd. for their support in the draft of this paper.

\section{References}

[1] R. Cervera, J. C. Piette, J. Font et al., "Antiphospholipid syndrome. Clinical and immunologic manifestations and pattern of disease expression in a cohort of 1,000 patients," Arthritis \& Rheumatology, vol. 46, no. 4, pp. 1019-1027, 2002.

[2] U. Picillo, S. Migliaresi, M. R. Marcialis, A. M. Ferruzzi, and G. Tirri, "Clinical significance of anticardiolipin antibodies in patients with systemic sclerosis," Autoimmunity, vol. 20, no. 1, pp. 1-7, 1995.

[3] R. Gupta, M. M. Thabah, S. Gupta, S. Shankar, and A. Kumar, "Clinical significance of antiphospholipid antibodies in Indian scleroderma patients," Rheumatology International, vol. 30, no. 2, pp. 277-279, 2009.
[4] M. Minatani, J. Takasaki, H. Iwata, M. Kinoshita, S. Aotsuka, and M. Sumiya, "Acute interstitial pneumonia associated with antiphospholipid syndrome in a patient with systemic sclerosis," Clinical and Experimental Rheumatology, vol. 18, article 786, no. 6, 2000.

[5] G. Zandman-Goddard, N. Tweezer-Zaks, T. Shalev, Y. Levy, M. Ehrenfeld, and P. Langevitz, "A novel overlap syndrome: systemic sclerosis associated with antiphospholipid syndrome-a case series," Annals of the New York Academy of Sciences, vol. 1108, pp. 497-504, 2007.

[6] W. H. Chun, D. Bang, and S. K. Lee, "Antiphospholipid syndrome associated with progressive systemic sclerosis," The Journal of Dermatology, vol. 23, no. 5, pp. 347-351, 1996.

[7] R. Whittaker, A. Barnett, and P. Ryan, "Antiphospholipid syndrome in scleroderma," Journal of Rheumatology, vol. 20, no. 9, pp. 1598-1600, 1993.

[8] M. Diallo, S. O. Niang, A. Kane, M. T. Dieng, and B. Ndiaye, "Antiphospholipid antibodies syndrome in dermatology in Dakar: 11 case report," Dakar Médical, vol. 52, no. 1, pp. 41-45, 2007.

[9] E. P. K. Yu, A. J. K. Ostor, and F. C. Hall, "Successful treatment with bosentan for severe digital ischaemia in limited cutaneous systemic sclerosis," Annals of the Rheumatic Diseases, vol. 66, no. 8, pp. 1122-1123, 2007.

[10] A. Ambach, W. Seo, B. Bonnekoh, and H. Gollnick, "Lowdose combination therapy of severe digital ulcers in diffuse progressive systemic sclerosis with the endothelin-1 receptor antagonist bosentan and the phosphodiesterase $\mathrm{v}$ inhibitor sildenafil," Journal of the German Society of Dermatology, vol. 7, no. 10, pp. 888-892, 2009. 


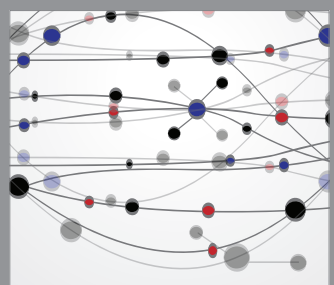

The Scientific World Journal
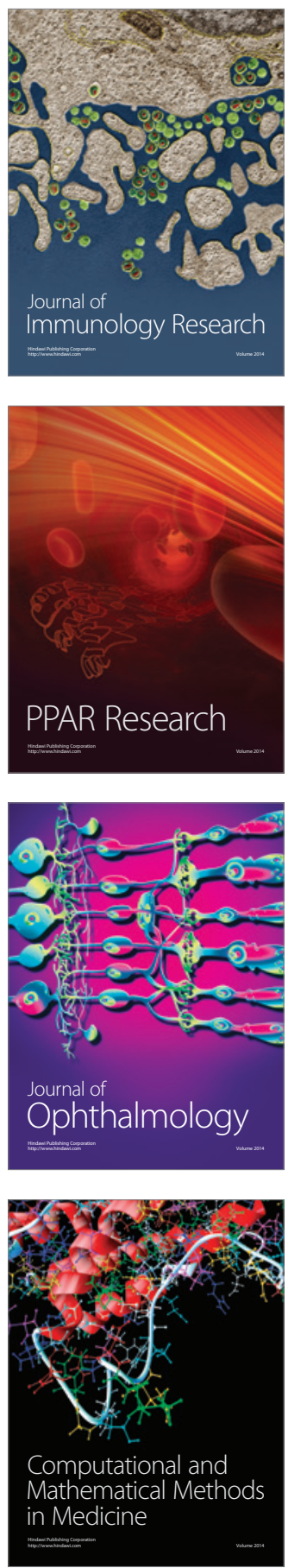

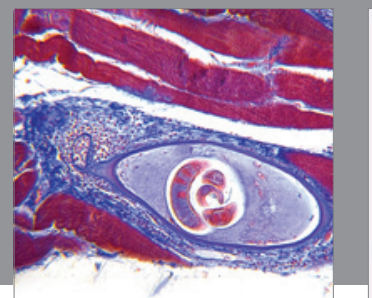

Gastroenterology

Research and Practice
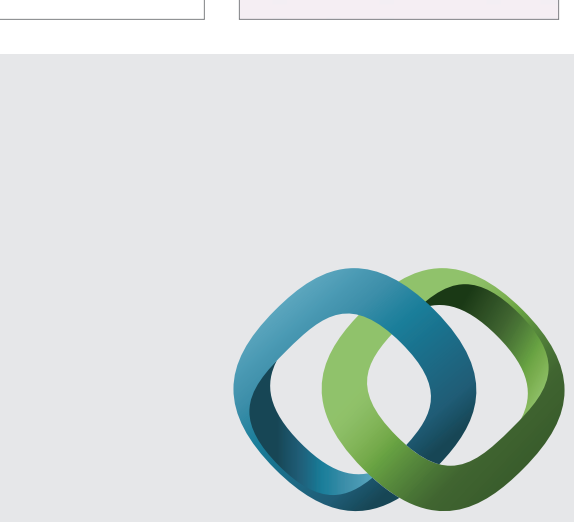

\section{Hindawi}

Submit your manuscripts at

http://www.hindawi.com
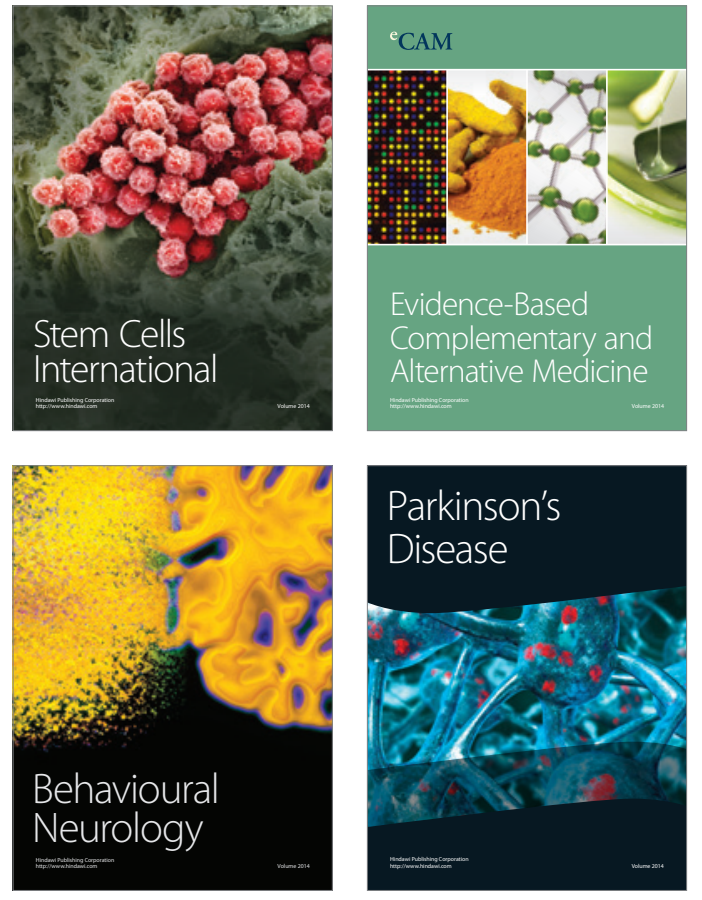
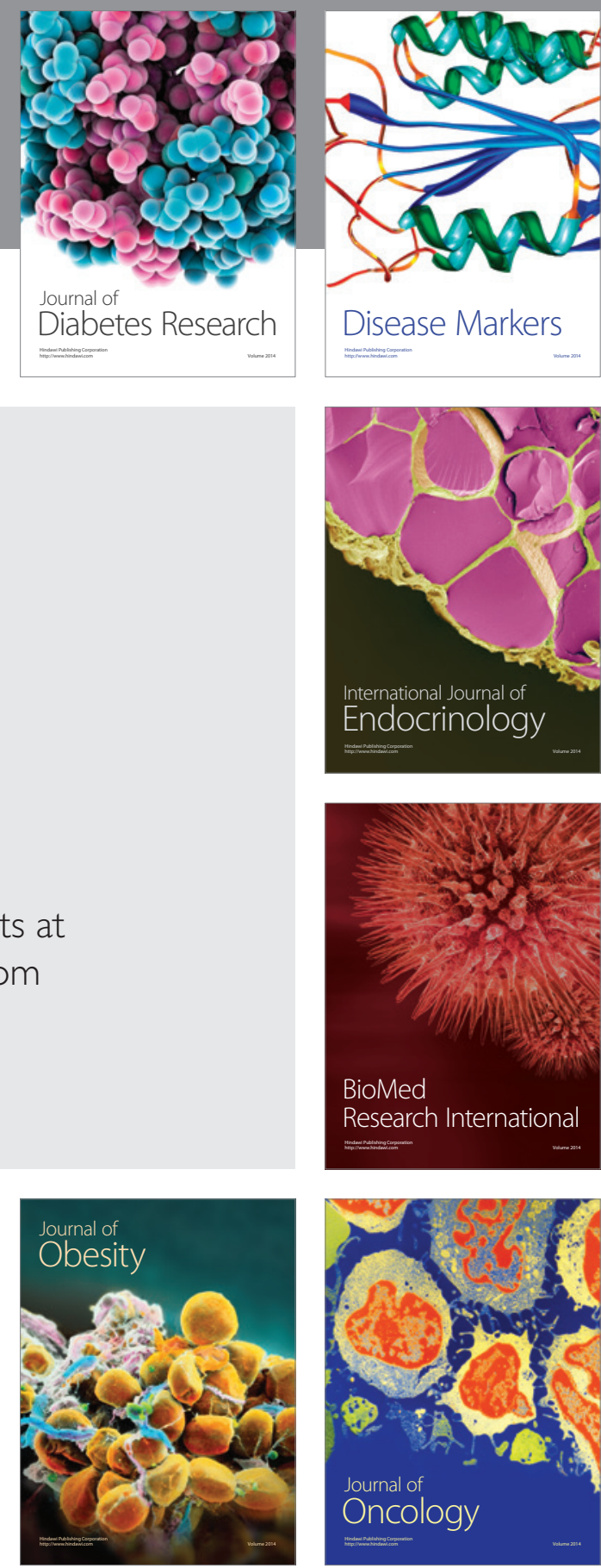

Disease Markers
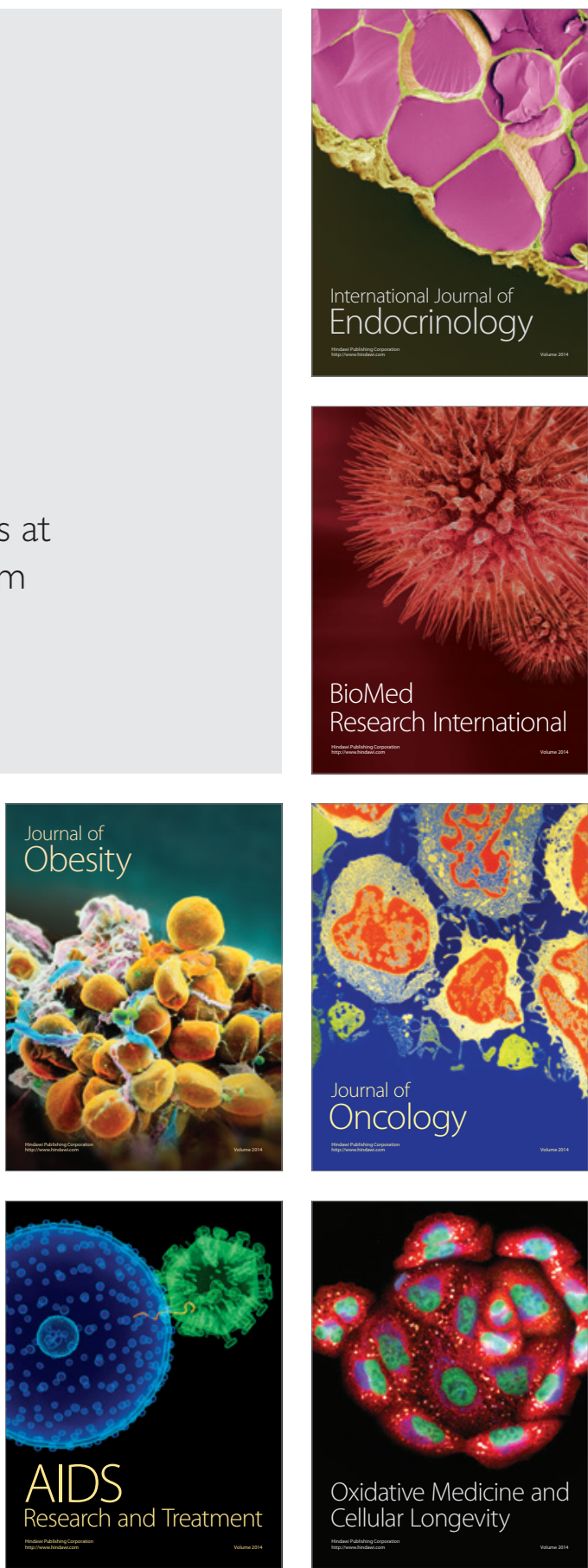